\section{São Gião de Nazaré (Portugal). Un tipo original de iglesia}

Luis Caballero, Fernando ArCe, M. a de los Ángeles Utrero Unidad Asociada CSIC/Universidad del País Vasco. Grupo de Arqueología Tardoantigua y Medieval. Arqueología de la Arquitectura (GATMAA)

\begin{abstract}
Resumen
La importancia científica de la iglesia portuguesa de São Gião de Nazaré, considerada como uno de los prototipos de la arquitectura visigoda peninsular del siglo vII, recomendaba un análisis arqueológico de su arquitectura complementario a la excavación del subsuelo y previo a los necesarios trabajos de restauración del edificio en amenaza de ruina. La lectura descubre una iglesia original de nave central con dos habitaciones altas a Oeste y Este, habitaciones laterales, iconostasis y ábside rectangular exento abovedado. Su estructura apenas varía hasta el momento de ruina y posterior transformación en casa labriega en el siglo XVIII. La adscripción cronológica de la iglesia, puesta en duda actualmente dentro de una problemática que afecta al conjunto de arquitectura tradicionalmente denominada visigoda, no se resuelve con la lectura, pero sí se enriquece con ella, al diferenciarse dos grupos decorativos, uno de piezas reutilizadas y otro de nueva talla. Este conjunto tallado ex profeso es ahora el indicador cronológico a seguir para fechar la iglesia original: para unos, de clara factura visigoda; para otros, asturiana.
\end{abstract}

Palabras clave: São Gião de Nazaré; análisis estratigráfico; arquitectura visigoda; arquitectura asturiana; reutilización.

\begin{abstract}
The scientific importance of the Portuguese church of São Gião de Nazaré, considered one of the prototypes of $7^{\text {th }}$ century lberian Visigoth architecture, merited an archaeological analysis of its architecture in addition to the excavation of its cellars and prior to necessary restoration work on the building, which was on the brink of ruin. Readings discovered an original church with a nave with high rooms to the west and east, side rooms, iconostases and freestanding vaulted rectangular apse. The structure scarcely changed until its ruin and subsequent conversion into farm workers' dwellings in the $18^{\text {th }}$ century.

The chronological ascription of the church, presently in doubt due to the problems concerning the architectural group traditionally called Visigoth, cannot be solved through the reading but can be enhanced by this as two different decorative groups are identified, one with reused pieces and the other with new sculptures. This deliberately sculpted decoration is now the chronological indicator to follow in dating the original church: for some, it is clearly Visigoth; for others, Asturian.
\end{abstract}

Key words: São Gião de Nazaré; stratigraphic analysis; Visigoth architecture; Asturian architecture; reuse.

\section{HISTORIOGRAFÍA: ¿VISIGODA O ASTURIANA?}

La lectura de paramentos de São Gião de Nazaré se planteó como instrumento de apoyo previo a la restauración y como herramienta de análisis de un edificio de relevante importancia histórica, historiográfica y monumental. Descubierta y parcialmente excavada a mediados de los años 60 (Almeida Y Borges, 1966), la iglesia de Nazaré fue incluida en el conjunto de iglesias visigodas del siglo VII (SCHLUnK, 1971), a pesar de poseer ciertos elementos, como la fábrica de mampostería o la tribuna superior a los pies de la nave, que no encajaban en las características que se suponían de este grupo. La presencia de estos elementos en ejemplos asturianos como San Julián de los Prados no es aceptada como paralelo, sino como consecuente del ejemplo portugués. La escultura decorativa, considerada como elaborada ex profeso y, por ello, contemporánea al edificio, determinaba el momento visigodo de la construcción.

Esta teoría ha estado vigente hasta momentos recientes, donde revisiones efectuadas desde diferentes enfoques, han introducido dudas en la teoría cimentada principalmente en el trabajo de SCHLUNK (1971) y continuada por su discípulo Arbeiter (1993). Como resultado del estudio de las técnicas constructivas, KINGSLEY (1980: 75-9, 143-5) opina que el aparejo de mampostería no puede fecharse antes del siglo IX. Ferreira de Almeida (1986: 136ss), en su revisión del arte mozárabe de Portugal, la data en el siglo X. Posteriormente, CABALLERO (1992) y ReAL (1995) proponen, con diferentes matices y combinando argumentos históricos y arqueológicos, una fecha dentro del IX o X, ya de época de Reconquista, en relación con la arquitectura prerrománica asturiana.

Parca en documentación escrita, como demuestra el informe histórico aportado por P. Penteado (1999), los trabajos arqueológicos se han retomado en la actualidad (L. FONTES), cuyos resultados no han visto todavía la luz.

Con esta problemática presente, la lectura perseguía la identificación de una secuencia estratigráfica y de aquellas partes originales del edificio. El precario estado de conservación de la iglesia, apuntalada en todo su perímetro exterior e interior, así como techada con una superficie metálica para defenderla de las humedades, impidió llevar a cabo una fotogrametría detallada, por lo que como base gráfica se adoptó aquella facilitada por el equipo de arquitectos, donde se mostraban las líneas maestras de la construcción. Todas estas estructuras de seguridad, así como los revocos que poseía el edificio en gran parte del interior dificultaron la realización del análisis estratigráfico en muchos puntos ocultos. Estos condicionantes deben tenerse en cuenta a la hora de valorar los resultados. 


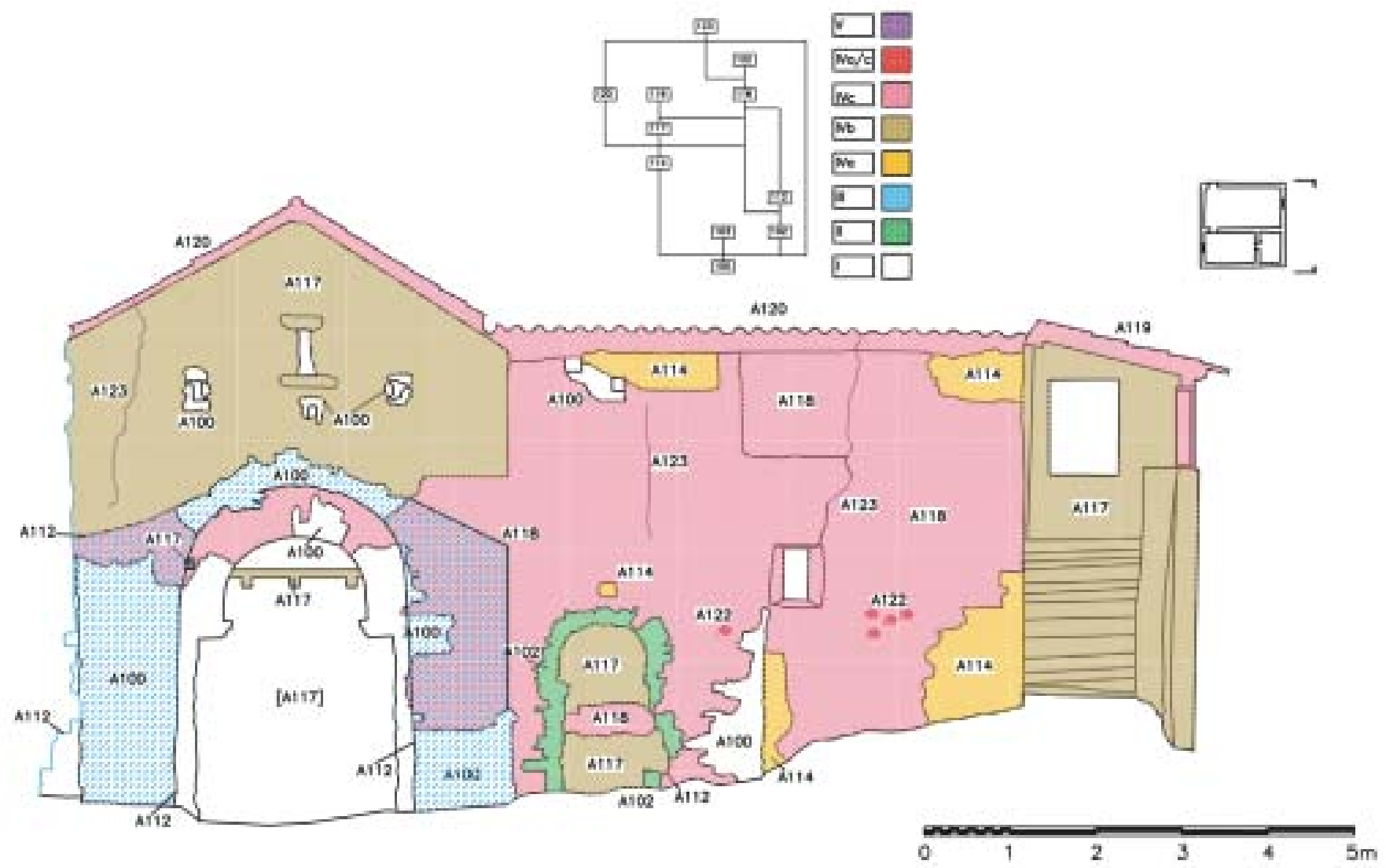

Fig. 1. Alzado este exterior

Descripción. La iglesia actual de São Gião de Nazaré se compone de una nave rectangular, rematada en un ábside del que sólo se conservan los arranques de los muros y de la bóveda, por lo que tanto la planta como el tipo de bóveda son desconocidas. El anteábside se separa del resto del aula por un muro horadado por dos huecos que flanquean un vano central, todos ellos rematados por arcos peraltados, $y$ se abre a los lados norte y sur por otros dos arcos respectivamente. Al norte se encuentra adosada una casa de dos pisos. El ala meridional de la iglesia, conocida por excavación y confirmada por la lectura, se encuentra actualmente perdida.

\section{SECUENCIA ESTRATIGRÁFICA:DE IGLESIA A CASA LABRIEGA}

Etapa I. Iglesia original. El análisis descubre una iglesia original unitaria (A100), como demuestra la unidad constructiva de los muros de la nave central y los testeros de la habitación norte, donde las relaciones estratigráficas y la técnica de aparejo confirman esta coetaneidad (Fig. 1 y 2). No se documenta, desde el análisis estratigráfico de los paramentos y frente a la opinión de REAL (1995), ninguna estructura anterior a la iglesia actual. En esta primera eta- pa, se leen nuevos elementos que ayudan a caracterizar la iglesia original.

Los restos de los muros laterales del ábside, del que no se conoce el testero oriental, muestran el inicio de unos nichos o arcos, hasta ahora no observados por la planimetría tradicional (SCHLUNK 1971). Los muros de la nave central poseen nichos altos en los paramentos que originalmente daban a las habitaciones laterales.

Respecto a las piezas decorativas, la lectura identifica dos grupos diferentes. Un primer grupo se compone de piezas antiguas reutilizadas en este momento, como ya había propuesto REAL (1995), entre las cuales situamos las impostas de origen romano del arco de triunfo que separa ábside y transepto y los cimacios y las columnas e imposta norte del arco septentrional de los arcos dobles del transepto. Un segundo grupo, de peor calidad y talla tosca, es realizado ex novo como imitación o retalle del anterior, donde citamos las impostas del arco de triunfo y las centrales del iconostasis. Este segundo grupo es ahora el indicador cronológico del edificio original.

Junto a la escultura arquitectónica, se documenta una decoración de doble círculo concéntrico con un punto central impreso sobre el estuco que cubre la zona sobre los ar- 

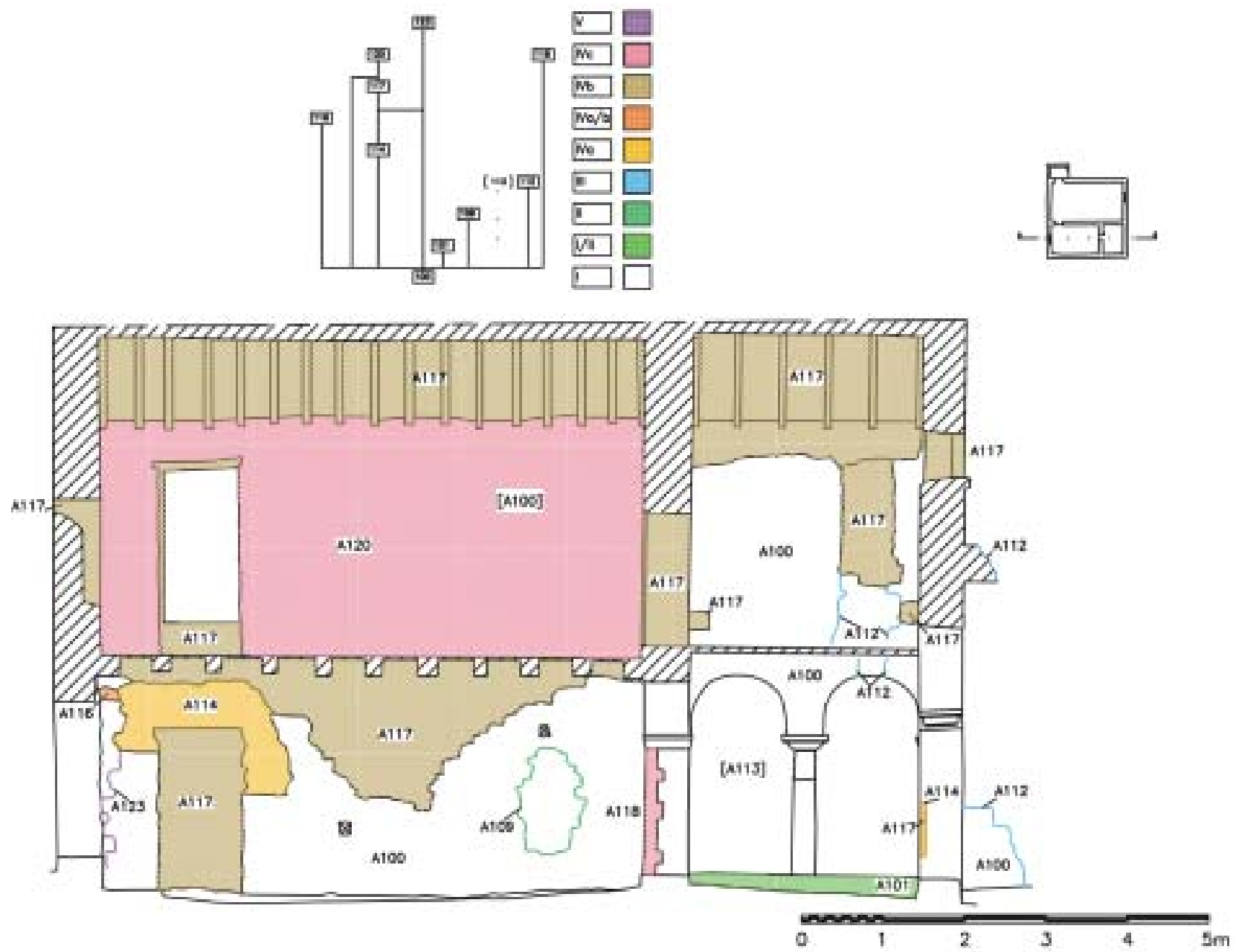

Fig. 2. Sección interior nave hacia el norte

cos dobles del muro sur del tramo central del transepto, a la altura del actual suelo del piso alto.

El análisis certifica la existencia de una tribuna occidental apoyada en dos ménsulas y dos vigas transversales. Junto a ésta, se descubre otra habitación alta sobre el transepto, donde se sitúan los restos de decoración de estuco, sostenida igualmente por ménsulas y vigas transversales, a la cual se accedía mediante una puerta ubicada en la esquina sudoeste. La puerta noroeste de la tribuna puede ser también original, pero los revocos no permiten afirmarlo con seguridad. Ambas habitaciones, la tribuna y la habitación oriental, estaban construidas en madera. En ninguna se ha documentado escalera alguna que facilitase el acceso a los vanos.

Etapa I/II. En una fase intermedia, se sitúa una serie de elementos cuya segura pertenencia al primer momento no es del todo certera. La relación del suelo de opus signinum (A101), que se adosa a muros y columnas, puede ser inter- pretada como etapa de obra o como relación de posterioridad. Lo mismo ocurre con el nicho cuadrado (A107) en la parte inferior del muro sur de la nave central.

Etapa II. Período de uso del edificio original. En un prolongado periodo de tiempo, desde la construcción original hasta su transformación en quinta agrícola en el siglo XVIII, se sucede una serie de cambios en el edificio de uso litúrgico. Muchas de estas transformaciones no tienen relación física directa entre sí, por lo que no se puede construir una secuencia diacrónica. Entre las actividades de este periodo, se encuentran la apertura de una puerta en el tramo norte del transepto (A102), reparaciones de suelos (A104 y 105), la desaparición de la habitación alta y de la tribuna occidental (A106), la reparación de los nichos originales (A108) y la apertura de otros nuevos (A109, A110).

Etapa III. Ruina del edificio original. En este momento tiene lugar la destrucción de las habitaciones laterales, el tra- 
mo sur del transepto y del cuerpo del ábside (A112). Las grietas de los muros apuntan al movimiento lateral de éstos como causa probable de la caída, pero no se puede afirmar cuál fue la causa de la destrucción y si ésta tuvo lugar de una sola vez o fue el resultado de un largo proceso.

Etapa IV. Casa labriega del siglo XVIII. Tras unos pequeños arreglos de conservación, la iglesia se convierte en una casa labriega. Esta etapa encierra dos fases diferentes. La primera transformación (A114) conlleva la prolongación de la fachada y del testero para unir a un nuevo muro de cierre septentrional, el de la iglesia queda englobado en la nueva estructura. De esta manera, el nuevo edificio comprende dos espacios: la antigua nave de la iglesia y el nuevo ámbito norte, comunicados por los arcos del transepto y la puerta oeste de la nave. En un segundo momento (A117), se dota a la casa de un segundo piso que requiere el alzado de un muro adosado al norte de la nave central de la iglesia para su solado. La obra conlleva además la construcción de una caja de escalera exterior y la readaptación de vanos y accesos. El uso de la casa da lugar a mejoras y reformas menores: muros adosados, suelos, enlucidos, aperturas y cierres de vanos, etc.

Etapa V. Siglo XX. Finalmente, el descubrimiento científico a mediados del siglo XX dejó una profunda huella material (A125): picado de muros, desmonte de tapiados y construcción de una caseta adosada al lado sur del transepto. El abandono se lee en las grietas (A123) y en los expolios modernos (A124) de material constructivo.

\section{LA IGLESIA ORIGINAL}

La lectura define una iglesia original formada por un cuerpo con armaduras de madera. El cuerpo se componía de una única nave separada con muros de las habitaciones norte y sur que la flanquean, rematada al este con un ábside exento abovedado, un transepto delimitado por un sistema de iconostasis, arcos peraltados y muros, una tribuna occidental y una habitación alta oriental en su tramo central. Elementos significativos son los nichos altos de las habitaciones y los arcos del ábside, todos ellos pertenecientes a un momento original. El hecho de que el aula principal se separe de las habitaciones septentrional y meridional, originalmente subdivididas en espacios menores, por muros corridos, nos hace hablar de una iglesia de única nave con transepto, diferenciado arquitectónicamente por un sistema de iconostasis, y ábside exento (Fig. 3 y 4).

La cronología del edificio, tradicionalmente basada en la tipología decorativa, sufre un cambio al confirmarse la reutilización de unas piezas y la talla ex profeso de otras, las cuales son ahora el indicador cronológico de este momento original.

\section{NUEVAS CUESTIONES: EL TIPO «ASTURIANO» Y LOS DOS GRUPOS DECORATIVOS}

Evaluación de resultados. Como ya hemos indicado, los obstáculos a los que se enfrentaba la lectura desde sus comienzos (enlucidos, andamiajes, cubiertas) han determinado en gran medida los resultados, los cuales han ofrecido nuevos datos, pero también nuevas dudas, las cuales podrían ser resueltas posiblemente con una revisión del edificio una vez realizada la restauración. Entre estas, señalaremos aquellas que consideramos fundamentales, no solamente para la comprensión de la evolución constructivo temporal, sino para la explicación de la funcionalidad del espacio en el momento original.

Litúrgicamente, la tribuna, la habitación alta y los nichos requieren una interpretación funcional así como la consecución de unos paralelos. Los accesos a la tribuna y a la habitación este se desconocen, no se ha constatado ninguna escalera o estructura que facilitase el acceso a estos vanos, por lo que podemos pensar en escaleras de madera. El enlucido tanto interior como exterior de la puerta norte impide conocer su momento secuencial, como ocurre igualmente en el vano sur. La contemporaneidad de ambos espacios, tribuna y habitación alta, incide en el problema del rito desarrollado en la iglesia. La hipótesis de SCHLUNK (1971) respecto a una iglesia conventual con espacios de uso diferenciados y jerarquizados arquitectónicamente necesita una revisión: aunque hallamos estos elementos en otras iglesias (iconostasis de Santullano y La Nave, tribuna de Quintanilla, Lillo, Lena o Valdediós, entre otros), no los encontramos combinados como en Nazaré. Respecto a los nichos, elementos conocidos en iglesias como Viguera, Siero y, de nuevo, en ejemplos asturianos como Nora o Santullano, su inusual ubicación en la parte alta de los paramentos que dan a los ámbitos norte y sur hace de los paralelos meras comparaciones formales, pero no funcionales.

La forma y dimensiones del ábside son desconocidas aunque, si tenemos en cuenta la planta casi cuadrada de nave y habitaciones (aprox. $1111 \mathrm{~m}$ ), se puede suponer la misma forma para el ábside siguiendo un esquema modular (ARIAS, 2001). La articulación en arcos embutidos de los muros laterales se completa con la cita de la presencia de una columna entre los arcos, como en los del transepto, en el documento de 1780 .

En la Etapa II, la imposibilidad de constatar relaciones directas entre las diferentes unidades estratigráficas no permite ofrecer una secuencia sincrónica de éstas, por lo que su posición estratigráfica no es definitiva. 
Nazaré y la arquitectura altomedieval. La constatación de esta serie de elementos implica necesariamente la contextualización de Nazaré en la arquitectura altomedieval peninsular, donde estas nuevas características amplían sin embargo la problemática que rodea a esta iglesia. Tipológicamente, la fábrica de mampostería, la planta de una nave con habitaciones laterales y transepto de tres tramos, los arcos peraltados, el sistema de cubriciones (ábside abovedado, nave con techumbre de madera), el iconostasis y la tribuna occidental no facilitan su inclusión en el grupo considerado visigodo del siglo VII. Muchos de estos elementos se encuentran, por el contrario, en la arquitectura del prerrománico asturiano del siglo IX.

Por otro lado, tampoco se puede desprender una cronología absoluta para este primer momento, donde, de nuevo, la tipología arquitectónica y decorativa vuelven a ser el instrumento de datación determinante, a la espera de los resultados que puedan ofrecer las nuevas excavaciones.

\section{Ficha técnica}

Instituto Portugués do Património Arquitectónico (IPPAR) - CSIC (Instituto de Historia).

Equipo, L. Caballero (Director), L. Fontes, M. Ramalho, M. da Cruz, F. Arce Sainz, C. Cauce, M. ${ }^{a}$ A. Utrero.

Enero 2001.

\section{Bibliografía}

Almeida F. DE, Borges García E., 1966, São Gião, descoberta e estudo arqueológico de um templo cristâo-visigótico na região de Nazaré, $\mathrm{Ar}$ queologia e Historia 8, serie 12, pp. 339-348 (con bibliografía anterior).

Arbeiter A., 1993, Baukunst und Kult in der Westgotenzeit. Die Kirche São Gião de Nazaré, Schubart H., Arbeiter A., Noack-Haley S., Funde in Portugal, Sternstunden der Archäologie, pp. 177-196, Göttingen-Zurich.

ArIas PÁrAmo, L. 2001, Fundamentos geométricos, metrológicos y sistemas de proporción en la arquitectura altomedieval asturiana (siglos IX y X), Archivo Español de Arqueología, 74, pp. 233-80.

Caballero Zoreda L., 1992, ¿Visigodo o Asturiano? Nuevos hallazgos en Mérida y otros datos para un nuevo marco de referencia de la arquitectura y la escultura altomedieval en el Norte y Oeste de la Península Ibérica, XXXIX Corso di Cultura sull'Arte Ravennate e Bizantina, pp. 139-90.

Ferreira de Almeida C.A., 1986, Arte Moçarabe e da Reconquista, História da Arte em Portugal, vol. II, Lisboa.

KIngsley K., 1980, Visigothic Architecture in Spain and Portugal: a Study in Masonry, Documents and Form, Berkley.

Penteado P., 1999, A igreja e a quinta de São Gião (Nazaré): fontes documentais para a sua história, Nazaré-Lisboa (manuscrito).

REAL M.L., 1995, Inovação e resistência: dados recentes sobre a antiguidade cristã no ocidente peninsular, IV Reunião de Arqueologia Cristã Hispânica (Lisboa 1992), pp. 17-68, Barcelona.

SCHlunK H., 1971, La Iglesia de São Gião de Nazaré. Contribución al estudio de la influencia de la liturgia en la arquitectura de las iglesias prerrománicas de la Península Ibérica, Actas do II Congresso Nacional de Arqueologia, vol. II, pp. 509-528, Coimbra. 